\title{
SPATIAL STIT TESSELLATIONS: DISTRIBUTIONAL RESULTS FOR I-SEGMENTS
}

\author{
CHRISTOPH THÄLE, ${ }^{*}$ Universität Osnabrück \\ VIOLA WEISS, ${ }^{* *}$ Fachhochschule Jena \\ WERNER NAGEL, ${ }^{* * *}$ Friedrich-Schiller-Universität Jena
}

\begin{abstract}
In this paper we consider three-dimensional random tessellations that are stable under iteration (STIT tessellations). STIT tessellations arise as a result of subsequent cell division, which implies that their cells are not face-to-face. The edges of the cell-dividing polygons are the so-called I-segments of the tessellation. The main result is an explicit formula for the distribution of the number of vertices in the relative interior of the typical I-segment. In preparation for its proof, we obtain other distributional identities for the typical I-segment and the length-weighted typical I-segment, which provide new insight into the spatiotemporal construction process.
\end{abstract}

Keywords: Cell division process; iteration/nesting; marked point process; random tessellation; stability under iteration; stochastic geometry

2010 Mathematics Subject Classification: Primary 60D05

Secondary 60G55; 60E05

\section{Introduction}

In recent years, random tessellation theory has been an active field of research. Whereas in the past mainly mean values and their relations were considered, current research focuses on second-order parameters, limit theorems, and distributional results; see [4], [5], and [6], among others. Besides the classical Poisson hyperplane and Poisson-Voronoi tessellations, random tessellations constructed by subsequent cell division have attracted particular interest in recent times in stochastic geometry and spatial statistics (see [1], [2], [14], and especially [3], and the references cited therein). Among these models, the so-called STIT tessellations (which are stable under iteration - see below) introduced in [9] and [12] are of particular interest, because of the number of analytically available results [7], [10], [13], [16]-[18], [20]-[23]; see Figure 1 for illustrations. The model shows the potential to become a new reference model for crack or fissure structures.

After detailed analyses of planar STIT tessellations in [10], [16], and [20], in this paper we consider the three-dimensional case. We study so-called I-segments in homogeneous threedimensional STIT tessellations, which form the one-dimensional building blocks of a STIT tessellation. They appear in the course of the sequential cell splitting procedure when cells are

\footnotetext{
Received 9 August 2011; revision received 11 December 2011.

* Postal address: Institut für Mathematik, Universität Osnabrück, Albrechtstr. 28a, D-49076 Osnabrück, Germany. Email address: christoph.thaele@uni-osnabrueck.de

** Postal address: Fachhochschule Jena, Fachbereich Grundlagenwissenschaften, Carl-Zeiss-Promenade 2, D-07745 Jena, Germany. Email address: viola.weiss@fh-jena.de

*** Postal address: Institut für Stochastik, Friedrich-Schiller-Universität Jena, Ernst-Abbe-Platz 2, D-07743 Jena, Germany. Email address: werner.nagel@uni-jena.de
} 

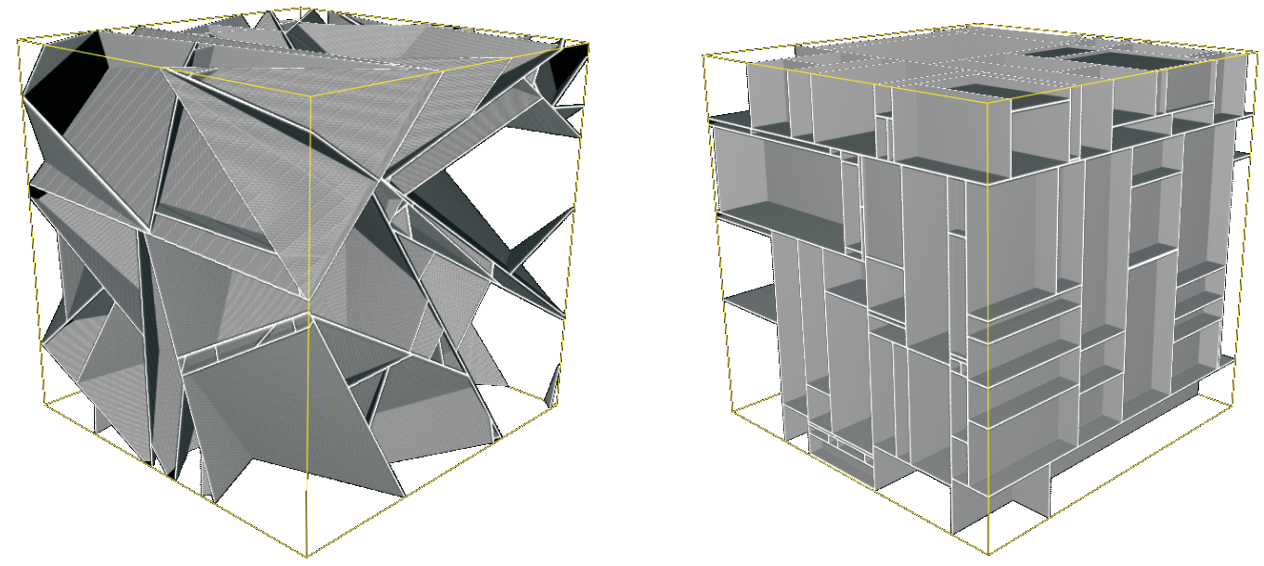

FIGURE 1: Realisations of an isotropic STIT tessellation (left) and a STIT tessellation whose directional distribution $\mathcal{R}$ is concentrated with equal weight on three orthogonal directions (right).
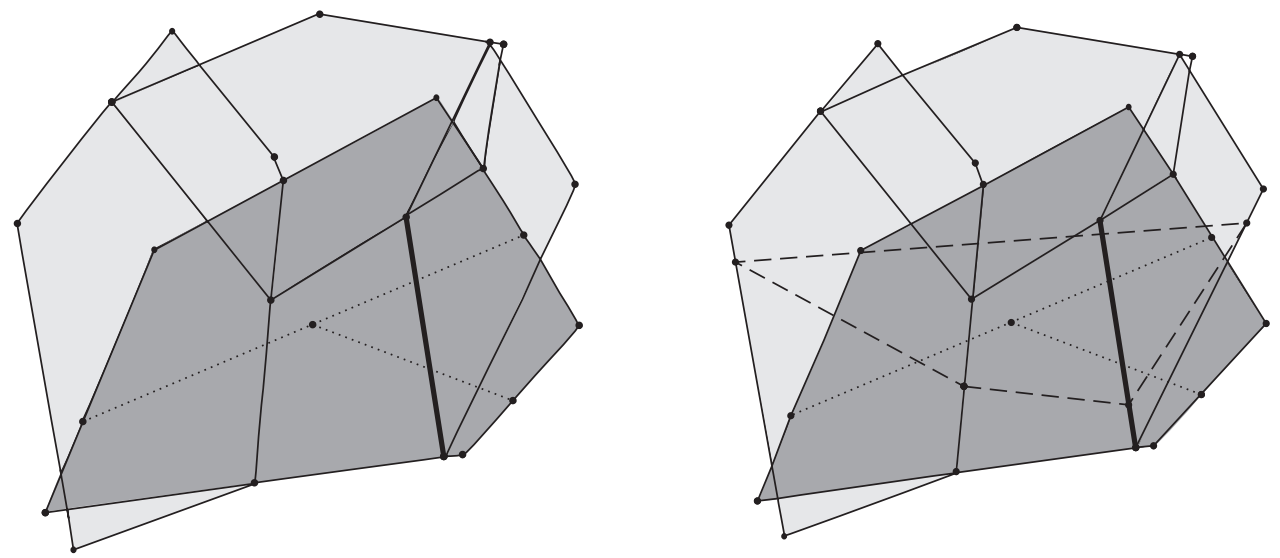

FIGURE 2: An I-segment (thick lines) together with its carrying I-polygon (dark-grey regions) at the time of its birth (left) and after further subdivision (right).

divided by new planes. In fact, all sides (1-faces) of a dividing two-dimensional polygonwhich will be referred to as an I-polygon - are called I-segments. In principle, the distribution of the number of vertices in the relative interior of the typical I-segment is the focus of this paper. Regarding the spatiotemporal construction, it becomes evident that this number depends on the birth time, the direction, and the length of the I-segment. But, it also depends on the birth time of that I-polygon, in whose interior the I-segment under consideration arises at a later time. This polygon is called the carrying I-polygon of the I-segment. At its birth time, this I-polygon is a facet of two adjacent mosaic cells, which, during the cell division process, undergo further subdivision (dashed polygon in Figure 2) and in this way new vertices and edges within the carrying I-polygon can appear; see Figure 2. For this reason, we consider the edges in the carrying I-polygon (dark-grey regions in Figure 2) and observe that an I-segment can have intersections with already existing edges (dotted lines in the dark-grey polygons in Figure 2) at the moment of its birth. This is in sharp contrast to the planar case studied by Mecke et al. [10] and Thäle [20]. In three dimensions the analysis is considerably more involved. 
Furthermore, vertices can arise after the birth of the typical I-segment as an effect of the ongoing cell division procedure. To derive an explicit formula for the probability that a fixed number of vertices is located in the relative interior of the typical I-segment, we first need to study the marked process of I-segments with the following marks: the length, direction and birth time of the I-segment, and the birth time of the carrying I-polygon. We will describe the joint and all marginal distributions of the marks associated to the typical and length-weighted typical I-segments of a homogeneous spatial STIT tessellation. Moreover, by noting that such a spatial tessellation has exactly two different types of vertices (see Figure 3 in Section 3) we can refine our result and obtain the joint distribution of the number of vertices of each type in the relative interior of the typical I-segment.

Note that it would also be interesting to calculate the distribution of the number of vertices within the typical two-dimensional building block, i.e. the typical I-polygon. However, this quantity is currently not accessible, because the area distribution of a typical Poisson polygon is needed. Determining this distribution is a long-standing open problem in stochastic geometry.

As already mentioned, related problems in the planar case have been studied in [10] and [20]. The results obtained there have led to a deeper understanding of planar STIT tessellations and have been used to obtain new results about their structure. In a companion paper [22] we also used the results of the present work to explore in more detail the combinatorial structure of spatial STIT tessellations, a study initiated in [21] and [23].

The paper is organized as follows. In Section 2 we introduce some notation, recall the basic construction of STIT tessellations, and rephrase some of their most important properties, which are needed for our later arguments. The conceptual framework as well as our main results are contained in Section 3. The proofs are the content of Section 4.

\section{STIT tessellations in $\mathbb{R}^{d}$}

In this section we explain our basic notation, the construction, and the main properties of STIT tessellations that are needed for our arguments below. Although our results are established for the three-dimensional case, we focus in this section on general space dimensions, because we apply some of the properties to lower-dimensional tessellations in our proofs.

\subsection{Notation}

A tessellation of $\mathbb{R}^{d}$ with $d \geq 1$ can be described in two ways: as a locally finite collection of nonoverlapping and space-filling compact convex polytopes (called cells in the sequel), as well as a closed subset of $\mathbb{R}^{d}$, which is formed by the union of all cell boundaries. We switch between both perspectives arbitrarily.

In this paper we will deal with random tessellations of $\mathbb{R}^{d}$, which can be regarded as random variables taking values in the measurable space of tessellations of $\mathbb{R}^{d}$; see [15] and [19] for a detailed definition and in particular for measurability issues. Our attention will be restricted to homogeneous (spatially stationary) random tessellations, i.e. random tessellations whose distributions are invariant under spatial translations.

We denote by $\mathscr{H}$ the set of all hyperplanes in $\mathbb{R}^{d}$. A hyperplane $h \in \mathcal{H}$ will be parametrized by its normal direction $u \in \delta_{+}^{d-1}$ (the upper unit half-sphere in $\mathbb{R}^{d}$ ) and its signed distance $p \in \mathbb{R}$ to the origin, where the distance is defined as positive if and only if the projection of the origin $o$ onto $h$ is located in the upper half-space. Such a hyperplane is denoted by $h=h(p, u) \in \mathcal{H}$. For $B \subset \mathbb{R}^{d}$, define

$$
[B]=\left\{(p, u) \in \mathbb{R} \times \varsigma_{+}^{d-1}: h(p, u) \cap B \neq \varnothing\right\}
$$


as the set of parameter values $(p, u)$ of hyperplanes $h(p, u)$ hitting the set $B$. Consider a measure $\Lambda$ on $\mathbb{R} \times \delta_{+}^{d-1}$ (equipped with the Borel product $\sigma$-field), which is the image under the described parametrization of a (nonzero) locally finite, translation invariant measure on $\mathcal{H}$. Invariance under translations implies that $\Lambda$ factorizes, i.e. there is a constant $\lambda>0$ and a probability measure $\mathcal{R}$ on $\ell_{+}^{d-1}$ with

$$
\Lambda=\lambda \mu \otimes \mathcal{R}
$$

where $\mu$ is the Lebesgue measure on $\mathbb{R}$. If $\mathcal{R}$ is the uniform distribution on $f_{+}^{d-1}$ and $\lambda=1$, then $\Lambda$ corresponds to the isometry-invariant hyperplane measure $\Lambda_{\text {iso }}$; cf. [15]. From now on we assume that $\lambda=1$ in factorization (1). Moreover, to avoid degenerate cases, we assume that $\mathcal{R}$ is not concentrated on a great half-subsphere of $\delta_{+}^{d-1}$, i.e. we require $\operatorname{span}(\operatorname{support}(\mathcal{R}))=\mathbb{R}^{d}$.

\subsection{Construction}

A formal and detailed description of STIT tessellations in bounded windows is given in [12]. Here we explain the construction in an intuitive way only. Let $\Lambda$ be a hyperplane measure as above, and let $W \subset \mathbb{R}^{d}$ be a bounded convex polytope. We assign to $W$ a random lifetime and on expiry of this lifetime we choose a random hyperplane, which splits $W$ into two polyhedral subcells $W^{+}$and $W^{-}$. The construction continues independently and recursively in both of the subcells $W^{+}$and $W^{-}$, which is to say that $W^{+}$and $W^{-}$are provided with independent random lifetimes and that they are divided by random hyperplanes when they die. Note that the hyperplanes are always chopped off by the boundary of their respective mother cells. This repeated cell division is continued until a fixed deterministic time threshold $t>0$ is reached. The random tessellation constructed until time $t$ within $W$ is denoted by $Y(t, W)$.

In order to ensure the temporal Markov property of the described cell splitting process, we assume that the lifetimes of the cells are conditionally (given the cells at a certain time) independent and exponentially distributed. Moreover, we assume that the parameter of this exponential lifetime distribution of a cell $c$ is given by $\Lambda([c])$ (and, thus, the parameters of the exponential distributions of different cells are not independent). In the special case $\Lambda=\Lambda_{\text {iso }}$, $\Lambda_{\text {iso }}([c])$ is proportional to the integral-geometric mean width of $c$. Note that this choice ensures that smaller cells live stochastically longer. Furthermore, we will assume that the hyperplane splitting a cell $c$ is chosen according to the law $\Lambda([c])^{-1} \Lambda(\cdot \cap[c])$.

\subsection{Important properties}

In this subsection we summarize the properties of STIT tessellations that are needed in our arguments below. For further background on STIT tessellations, we refer the reader to [9] and [12], and to [15] and [19], for a general introduction to stochastic geometry.

If we insert independent copies $Y_{c}(s)$ of the tessellation $Y(s)$ into the cells $c$ of $Y(t)$ (generating $Y_{c}(s) \cap c$ ), the resulting iterated or nested tessellation is denoted by

$$
Y(t) \boxplus Y(s)=Y(t) \cup \bigcup_{c \text { a cell of } Y(t)}\left(Y_{c}(s) \cap c\right) .
$$

Considered as a random closed set, $Y(t, W)$ satisfies the following property.

(P1) (Spatial consistency) The random tessellation $Y(t, W)$ is spatially consistent in that, for any convex $V \subset W$ with $W$ as in Subsection 2.1, we have $Y(t, W) \cap V \stackrel{\mathrm{D}}{=} Y(t, V)$ (where 'D ' stands for equality in distribution). Thus, Kolmogorov's extension theorem ensures that there exists a homogeneous random tessellation $Y(t)$ in the whole $\mathbb{R}^{d}$ satisfying 
$Y(t) \cap W \stackrel{\mathrm{D}}{=} Y(t, W)$. For the particular choice $\Lambda=\Lambda_{\text {iso }}, Y(t)$ is also isotropic, i.e. its distribution is rotation invariant.

In [9] a global construction of $Y(t)$ is provided, where the spatiotemporal random process $(Y(t))_{t>0}$ is defined. Its important properties are now summarized.

(P2) (Scaling) The distributions of the rescaled tessellations are identical, i.e. $t Y(t) \stackrel{\mathrm{D}}{=} s Y(s)$ for all $s, t>0$.

(P3) (Iteration stability) We have

$$
Y(t) \stackrel{\mathrm{D}}{=} Y(s) \boxplus Y(t-s), \quad 0<s<t,
$$

where $Y(s)$ and $Y(t-s)$ are independent. Consequently, owing to (P2),

$$
Y(t) \stackrel{\mathrm{D}}{=} n Y(n t) \stackrel{\mathrm{D}}{=} n(\underbrace{Y(t) \boxplus \cdots \boxplus Y(t)}_{n \text { times }})
$$

for $t>0$ and $n \in \mathbb{N}$. The latter relation is usually referred to as stability under iterations. For this reason, the random tessellations $Y(t)$ are called STIT tessellations.

(P4) (Poisson typical cell) The distribution of the interior of the typical cell of $Y(t)$ coincides with that of the interior of the typical cell of a homogeneous Poisson hyperplane tessellation with intensity measure $t \Lambda$ (cf. [15] and [19]).

(P5) (Interpretation of $t$ and $\mathcal{R})$ The surface density $S_{V}$ of $Y(t)$, that is, the mean total $(d-1)$ volume of cell boundaries of $Y(t)$ per unit $d$-volume, is equal to the construction time $t>0$, i.e. $S_{V}=t$. The probability measure $\mathcal{R}$, see (1), is the distribution of the normal direction in the typical boundary point of $Y(t)$, called the directional distribution of the tessellation (that is, the surface-area-weighted directional distribution of the cell boundaries).

(P6) (Linear sections) The intersection of $Y(t)$ with a line $L$ induces a homogeneous Poisson point process on $L$ with intensity $\Lambda([e(L)]) t$, where $e(L)$ is a segment of unit length on $L$.

(P7) (Temporal Markov property) The random process $(Y(t))_{t>0}$ with values in the space of tessellations satisfies the Markov property in time.

\section{Framework and results}

In this section we present our main results, Theorem 1, Theorem 2, and Theorem 3, and their corollaries. Before we can state them, we have to introduce further notation and the framework of our work.

\subsection{The marked process of I-segments in spatial STIT tessellations}

The notion of I-segments is due to R. Miles and characterizes one of the types of line segment associated with a random planar tessellation that is not face-to-face; cf. [11] and the references cited therein. An I-segment can be defined as a maximal union of collinear and connected line segments that appear in the edge skeleton of a tessellation. Obviously, this definition does not depend on the dimension of the ambient space and will henceforth also be used for the spatial case, $d=3$. Generalizing Miles' concept, an I-polygon is a maximal union of coplanar 
and convex planar polygons (subsets of a plane in $\mathbb{R}^{3}$ ). Here, 'maximal' is understood in the sense that there is no extension to a larger collinear (coplanar) and convex set in the tessellation (considered as the closed set of cell boundaries).

In terms of the spatiotemporal construction of STIT tessellations $Y(t)$, the I-segments and I-polygons in spatial STIT tessellations can be characterized as follows. Any I-polygon of $Y(t)$ is a cell-dividing planar polygon introduced until time $t$, and any I-segment is a side (1-face) of an I-polygon. When a cell $c$ is divided by a plane $h \in[c]$, exactly one I-polygon $c \cap h$ is born, whereas at least three I-segments are born simultaneously. Any of these I-segments is the intersection of the I-polygon $c \cap h$ with a facet (2-face) of the cell $c$. This facet itself is embedded in a (possibly larger) previously born I-polygon, which is called the carrying I-polygon of the I-segment.

For our purposes, it will be appropriate to describe the joint distribution of the following marks associated to an I-segment in $Y(t)$ :

$$
\left(\ell, \varphi, \beta, \beta_{\text {carr }}\right) \in(0, \infty) \times 8_{+}^{2} \times(0, t) \times(0, t) .
$$

Here $\ell, \varphi$, and $\beta$ are the length, direction, and the birth time of the I-segment, respectively, and $\beta_{\text {carr }}$ is the birth time of the carrying I-polygon. Note that the direction of a segment is the unique unit vector in $\delta_{+}^{2}$ parallel to the segment.

Consider for fixed time $t>0$ and measure $\Lambda$ the homogeneous STIT tessellation $Y(t)$. Then the process of the marked I-segments of $Y(t)$ with marks as in (3) is a homogeneous marked segment process. Thus, Palm calculus for marked point processes can be applied and allows us to define the distribution of the typical I-segment and its mark distribution; see [15], [19], and also Section 4 below. In intuitive terms, the typical I-segment and its mark distribution can be regarded as a randomly (equally likely) chosen I-segment of $Y(t, W)$ together with its random marks when $W$ is a 'large' observation window.

In the following we use the indicator function notation $\mathbf{1}\{\cdot\}$, which is 1 if the statement in brackets is fulfilled and 0 otherwise. Let us also define the two constants

$$
\begin{aligned}
\zeta_{2} & :=\int_{\mathcal{S}_{+}^{2}} \int_{\mathcal{S}_{+}^{2}}[u, v] \mathcal{R}(\mathrm{d} u) \mathcal{R}(\mathrm{d} v), \\
\zeta_{3} & :=\int_{\delta_{+}^{2}} \int_{\delta_{+}^{2}} \int_{\delta_{+}^{2}}[u, v, w] \mathcal{R}(\mathrm{d} u) \mathcal{R}(\mathrm{d} v) \mathcal{R}(\mathrm{d} w),
\end{aligned}
$$

where $[u, v]$ is the area of the parallelogram spanned by $u, v \in \delta_{+}^{2}$ and $[u, v, w]$ stands for the volume of the parallelepiped spanned by $u, v, w \in \delta_{+}^{2}$ (interpreted as unit vectors in $\mathbb{R}^{3}$ having one endpoint at the origin). Note that in the isotropic case these constants are given by $\zeta_{2}=\pi / 4$ and $\zeta_{3}=\pi / 8$. We further introduce the following probability distributions on $8_{+}^{2}$ :

$$
\begin{aligned}
\widetilde{R}(U) & :=\frac{1}{\zeta_{2}} \int_{\delta_{+}^{2}} \int_{\delta_{+}^{2}} 1\left\{u^{\perp} \cap v^{\perp} \cap \delta_{+}^{2} \in U\right\}[u, v] \mathcal{R}(\mathrm{d} u) \mathcal{R}(\mathrm{d} v), \\
\mathcal{R}_{\mathrm{typ}}(U) & :=\frac{\zeta_{2}}{\zeta_{3}} \int_{\delta_{+}^{2}} \mathbf{1}\{u \in U\} \Lambda([u]) \widetilde{\mathcal{R}}(\mathrm{d} u) .
\end{aligned}
$$

Here $U \subset \delta_{+}^{2}$ is a Borel set, $\Lambda([u])$ stands for the $\Lambda$-measure of the set of all planes hitting the unit line segment connecting $u \in \delta_{+}^{2} \subset \mathbb{R}^{3}$ with the origin $o$, and $u^{\perp}$ denotes the orthogonal complement of $u \in 8_{+}^{2}$. For a Poisson plane tessellation with intensity measure $\Lambda$, the distributions $\widetilde{\mathcal{R}}$ and $\mathcal{R}_{\text {typ }}$ are the length-weighted distribution of the direction of edges and the directional distribution of the typical edge, respectively; see [6]. In the particular isotropic case we have $\Lambda([u])=\frac{1}{2}$ for any $u \in \delta_{+}^{2}$, and, thus, $\widetilde{\mathcal{R}}$ and $\mathcal{R}_{\text {typ }}$ are the uniform distributions on $\delta_{+}^{2}$. 


\subsection{Statement of results}

We are now in a position to present the main results of this paper. We start with a description of the distribution of marks, see (3), associated with the typical I-segment.

Theorem 1. Let $Y(t)$ be a homogeneous random STIT tessellation in $\mathbb{R}^{3}$ with measure $\Lambda$ as in (1).

(i) The distribution of the direction of the typical I-segment of $Y(t)$ equals $\mathcal{R}_{\text {typ }}$ as defined in (6).

(ii) The joint birth time density of $\left(\beta, \beta_{\text {carr }}\right)$ of the typical I-segment of $Y(t)$ equals

$$
p_{\beta, \beta_{\text {carr }}}(s, r)=\frac{3 s}{t^{3}} \mathbf{1}\{0<r<s<t\} .
$$

(iii) The conditional length density of the typical I-segment of the STIT tessellation $Y(t)$, given $\left(\varphi, \beta, \beta_{\text {carr }}\right)=(u, s, r) \in s_{+}^{2} \times(0, t)^{2}$ with $0<r<s<t$, is

$$
p_{\ell \mid \varphi=u, \beta=s, \beta_{\text {carr }}=r}(x)=\Lambda([u]) s \mathrm{e}^{-\Lambda([u]) s x} \mathbf{1}\{x>0\} .
$$

It is interesting to note that the conditional length density in Theorem 1(iii) does not depend on $r$ and that the joint distribution of the birth time vector $\left(\beta, \beta_{\text {carr }}\right)$ in part (ii) is independent of $\Lambda$ (and also of $\mathcal{R})$.

Now we derive some consequences of Theorem 1. First, we calculate some marginal and conditional birth time densities.

Corollary 1. For the homogeneous random STIT tessellation $Y(t)$, we have

$$
\begin{aligned}
p_{\beta_{\mathrm{carr}}}(r) & =\frac{3}{2} \frac{t^{2}-r^{2}}{t^{3}} \mathbf{1}\{0<r<t\}, \\
p_{\beta}(s) & =\frac{3 s^{2}}{t^{3}} \mathbf{1}\{0<s<t\} .
\end{aligned}
$$

Moreover,

$$
p_{\beta \mid \beta_{\text {carr }}=r}(s)=\frac{2 s}{t^{2}-r^{2}} \mathbf{1}\{0<r<s<t\}, \quad p_{\beta_{\text {carr }} \mid \beta=s}(r)=\frac{1}{s} \mathbf{1}\{0<r<s\},
$$

i.e. the conditional birth time distribution of the carrying I-polygon, given that the I-segment is born at time $0<s<t$, is the uniform distribution on $(0, s)$.

Using Theorem 1(ii) and (iii), we obtain the following result.

Corollary 2. The direction $\varphi$ and the birth time pair $\left(\beta, \beta_{\text {carr }}\right)$ of the typical I-segment of $Y(t)$ are independent. Moreover, the joint conditional density of the length and birth times, given its segment direction $u \in \diamond_{+}^{2}$, is

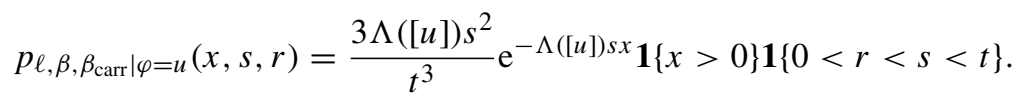

Integration now yields the marginal density for the length of the typical I-segment of the STIT tessellation $Y(t)$. This density is already known from [18]; see also Lemma 5 below. 
Corollary 3. The length density of the typical I-segment of $Y(t)$ equals

$$
\begin{aligned}
p_{\ell}(x)=\int_{\delta_{+}^{2}} \frac{3}{\Lambda([u])^{3} t^{3} x^{4}} & \left(6-\left(6+6 \Lambda([u]) t x+3 \Lambda([u])^{2} t^{2} x^{2}\right.\right. \\
& \left.\left.+\Lambda([u])^{3} t^{3} x^{3}\right) \mathrm{e}^{-\Lambda([u]) t x}\right) \mathcal{R}_{\mathrm{typ}}(\mathrm{d} u), \quad x>0 .
\end{aligned}
$$

In the isotropic case this reduces to

$$
p_{\ell}(x)=\frac{3}{t^{3} x^{4}}\left(48-\left(48+24 t x+6 x^{2} t^{2}+t^{3} x^{3}\right) \mathrm{e}^{-t x / 2}, \quad x>0 .\right.
$$

Now we turn to the distribution of the number of vertices in the relative interior of the typical I-segment. In fact, with Theorem 1 we obtain the following result.

Theorem 2. The probability $\mathrm{p}_{n}$ that the typical I-segment of $Y(t)$ has exactly $n \in \mathbb{N}$ vertices in its relative interior is given by

$$
\mathrm{p}_{n}=3 \int_{0}^{1} \int_{0}^{1}(1-a)^{3} \frac{(3-(1-a)(3-b))^{n}}{(3-(1-a)(2-b))^{n+1}} \mathrm{~d} b \mathrm{~d} a .
$$

It is interesting to note that the distribution in Theorem 2 does not depend on the measure $\Lambda$ (or, equivalently, the directional distribution $\mathcal{R}$ ) and the time parameter $t$. But, this is evident, because the number of vertices in the relative interior of the typical I-segment does not change when the tessellation is scaled in space. However, the latter is, owing to (P2), equivalent to a rescaling of time. Some particular values for $\mathrm{p}_{n}$ are summarized in Table 1.

From (8), we conclude the following result.

Corollary 4. The mean number of vertices in the relative interior of the typical I-segment is equal to 2. Moreover, the variance equals $\frac{59}{3}$ and all higher moments of that random variable are infinite.

Note that the mean value is in accordance with the result in [21]. Furthermore, the nonexistence of higher moments is not surprising, because moments of order greater than or equal to 3 of the length of the typical I-segment of $Y(t)$ are also infinite.

As a last result, we would like to point out that Theorem 2 admits a refinement, which is needed in [22]. We first note that a spatial STIT tessellation has exactly two different types of vertices, T-vertices and X-vertices; see [21]-[23]. Illustrations of these two types of vertices are shown in Figure 3. Given a carrying I-polygon (dark-grey regions in Figure 3), a T-vertex is generated if two further I-polygons intersect in the same half-space determined by the carrying I-polygon. An X-vertex is generated by an intersection of two further polygons in the two different half-spaces specified by the carrying I-polygon.

For the typical I-segment, we denote by $\mathrm{p}_{m, n}, m, n \in \mathbb{N}$, the probability that it has exactly $m$ vertices of type $\mathrm{T}$ and $n$ vertices of type $\mathrm{X}$ in its relative interior.

TABLE 1.

\begin{tabular}{ccc}
\hline$n$ & $\mathrm{p}_{n}$ (exact value) & $\mathrm{p}_{n}$ (numerical value) \\
\hline 0 & $\frac{189}{8} \ln 3-26 \ln 2-\frac{15}{2}$ & 0.43289 \\
1 & $\frac{1593}{16} \ln 3-107 \ln 2-35$ & 0.21384 \\
2 & $\frac{5319}{16} \ln 3-350 \ln 2-\frac{245}{2}$ & 0.11841 \\
3 & $\frac{31617}{32} \ln 3-1025 \ln 2-\frac{4499}{12}$ & 0.07075 \\
\hline
\end{tabular}



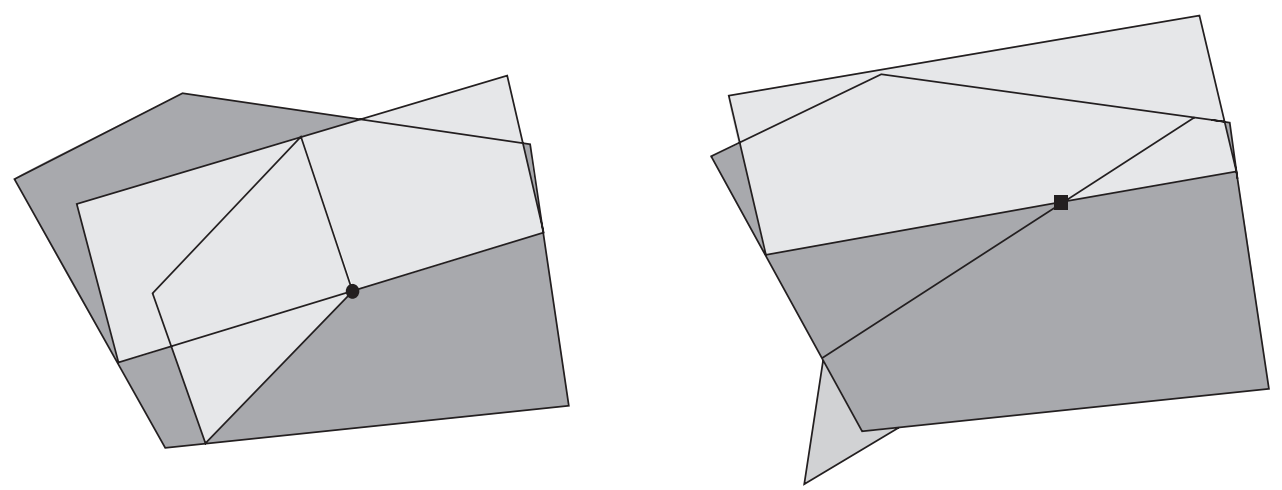

FIGURE 3: A T-vertex (left) and an X-vertex (right) in a spatial STIT tessellation.

Theorem 3. For a homogeneous spatial STIT tessellation and $m, n \in \mathbb{N}$, we have

$$
\mathrm{p}_{m, n}=3 \cdot 2^{m}\left(\begin{array}{c}
m+n \\
m
\end{array}\right) \int_{0}^{1} \int_{0}^{1}(1-a)^{3} a^{m} \frac{(1-(1-a)(1-b))^{n}}{(3-(1-a)(2-b))^{m+n+1}} \mathrm{~d} b \mathrm{~d} a .
$$

From this formula, the following can be concluded.

Corollary 5. The mean numbers of T-vertices and X-vertices in the relative interior of the typical I-segment equal 1. Furthermore, the variance of the number of T-type vertices is 8 and the variance of the number of X-type vertices is $\frac{11}{3}$ (all higher moments are infinite). In addition, the covariance of the number of $T$ - and $X$-vertices equals 4.

In [21] and [23] it was shown that the proportion of the intensities of T- and X-vertices in a spatial STIT tessellation is $2: 1$. Whereas any T-vertex is located in the relative interior of exactly one I-segment, an X-vertex is in the relative interior of two I-segments. This confirms the first statement of Corollary 5.

\section{Proofs}

Before proving our results for the typical I-segment, we first consider the mark distribution in a typical edge point, i.e. the length-weighted mark distribution. The corresponding results are derived in Subsection 4.2. The proofs of Theorems 1-3 and their corollaries are the content of Subsection 4.3. Some preparatorial material is collected in Subsection 4.1.

\subsection{Some preparations}

We denote by $\mathcal{L}$ the measurable space of line segments in $\mathbb{R}^{3}$, and equip it with the Borel $\sigma$-field induced by the Hausdorff distance; cf. [15]. The distribution on $\mathcal{L}$ of the typical I-segment of a STIT tessellation $Y(t)$ for fixed $t>0$ and fixed measure $\Lambda$ is denoted by $\mathbb{D}^{Y(t)}$, and, similarly, the distribution of the typical edge of a Poisson plane tessellation with intensity measure $s \Lambda$ is denoted by $\mathbb{D}^{P(s)}$.

In Theorem 3 of [18] the following is shown.

Lemma 1. For any nonnegative measurable function $f: \mathcal{L} \rightarrow \mathbb{R}$, we have

$$
\int f(L) \mathbb{D}^{Y(t)}(\mathrm{d} L)=\int_{0}^{t} \int \frac{3 s^{2}}{t^{3}} f(L) \mathbb{D}^{P(s)}(\mathrm{d} L) \mathrm{d} s .
$$


In order to apply this lemma, we will need the length distribution of the edges in Poisson plane tessellations. We consider the survival function of the conditional length distribution of the typical edge of $P(s)$, given its direction $u \in \varsigma_{+}^{2}$, i.e.

$$
H_{\ell \mid \varphi=u}^{P(s)}(x)=\int \mathbf{1}\{\operatorname{length}(L)>x\} \mathbb{D}_{\varphi(L)=u}^{P(s)}(\mathrm{d} L),
$$

where $\mathbb{D}_{\varphi(L)=u}^{P(s)}(\mathrm{d} L)$ denotes the respective conditional distribution of the typical edge. The length-weighted case $\widetilde{H}_{\ell \mid \varphi=u}^{P(s)}$ is defined analogously. In the following we will always use a tilde to indicate that we refer to a length-weighted distribution.

Lemma 2. The survival function of the conditional length distribution of the typical edge in the Poisson plane tessellation $P(s)$ is given by

$$
H_{\ell \mid \varphi=u}^{P(s)}(x)=\mathrm{e}^{-\Lambda([u]) s x} \text { for } x>0,
$$

and the survival function of the conditional length distribution of the length-weighted typical edge in the Poisson plane tessellation $P(s)$ is given by

$$
\widetilde{H}_{\ell \mid \varphi=u}^{P(s)}(x)=(1+\Lambda([u]) s x) \mathrm{e}^{-\Lambda([u]) s x} \text { for } x>0 .
$$

Proof. The intersection of the homogeneous Poisson plane process $P(s)$ with a fixed plane parallel to $u \in 8_{+}^{2}$ is a Poisson line process. The intersection of this line process with a fixed line parallel to $u$ is a Poisson point process with intensity $\Lambda([u]) s$; see, e.g. [15, Theorem 4.4.6] or, more specifically, Equation (4.31) ibidem. Thus, it follows from a twofold application of Slivnyak's theorem for Poisson processes that the typical edge point of a Poisson plane tessellation is almost surely located on a length-weighted segment that is generated by a linear homogeneous Poisson point process, and, under the condition that the direction is $u$, its intensity is $\Lambda([u]) s$. Hence, the length of the typical edge, under the condition $\varphi=u$, is exponentially distributed with parameter $\Lambda([u]) s$, and the respective length-weighted distribution is the gamma distribution with parameters $(2, \Lambda([u]) s)$. This completes the proof.

As a corollary, we obtain the mean length of the typical edge in a Poisson plane tessellation.

Lemma 3. The mean length of the typical edge in the Poisson plane tessellation $P(s)$ is

$$
\bar{\ell}^{P(s)}=\frac{1}{s} \frac{\zeta_{2}}{\zeta_{3}} .
$$

Proof. Using Theorem 1 of [6], which says that $\mathcal{R}_{\text {typ }}$ is the directional distribution of the typical edge in a Poisson plane tessellation, and (6), we obtain

$$
\begin{aligned}
\bar{\ell}^{P(s)} & =\int_{0}^{\infty} H_{\ell}^{P(s)}(x) \mathrm{d} x \\
& =\int_{0}^{\infty} \int_{\delta_{+}^{2}} H_{\ell \mid \varphi=u}^{P(s)}(x) \mathcal{R}_{\mathrm{typ}}(\mathrm{d} u) \mathrm{d} x \\
& =\frac{\zeta_{2}}{\zeta_{3}} \int_{\delta_{+}^{2}} \int_{0}^{\infty} \mathrm{e}^{-\Lambda([u]) s x} \Lambda([u]) \mathrm{d} x \widetilde{\mathcal{R}}(\mathrm{d} u) \\
& =\frac{1}{s} \frac{\zeta_{2}}{\zeta_{3}},
\end{aligned}
$$

which proves our claim. 
We now derive from Lemma 1 a similar representation for the distribution $\widetilde{\mathbb{D}}^{Y(t)}$ of the lengthweighted typical I-segment of $Y(t)$ in terms of $\widetilde{\mathbb{D}}^{P(s)}$, the distribution of the length-weighted typical edge of a Poisson plane tessellation.

Lemma 4. For any nonnegative measurable function $f: \mathcal{L} \rightarrow \mathbb{R}$, we have

$$
\int f(L) \widetilde{\mathbb{D}}^{Y(t)}(\mathrm{d} L)=\int_{0}^{t} \int \frac{2 s}{t^{2}} f(L) \widetilde{\mathbb{D}}^{P(s)}(\mathrm{d} L) \mathrm{d} s .
$$

Proof. Denoting the mean length of the typical I-segment of $Y(t)$ by $\bar{\ell}^{t}$ and that of the typical edge of a Poisson plane tessellation $P(s)$ by $\bar{\ell}^{P(s)}$, we have

$$
\begin{aligned}
\int f(L) \widetilde{\mathbb{D}}^{Y(t)}(\mathrm{d} L) & =\frac{1}{\bar{\ell}^{t}} \int f(L) \text { length }(L) \mathbb{D}^{Y(t)}(\mathrm{d} L) \\
& =\frac{1}{\bar{\ell}^{t}} \int_{0}^{t} \int \frac{3 s^{2}}{t^{3}} f(L) \operatorname{length}(L) \mathbb{D}^{P(s)}(\mathrm{d} L) \mathrm{d} s \\
& =\frac{1}{\bar{\ell}^{t}} \int_{0}^{t} \frac{3 s^{2}}{t^{3}} \bar{\ell}^{P(s)}\left(\int f(L) \tilde{\mathbb{D}}^{P(s)}(\mathrm{d} L)\right) \mathrm{d} s
\end{aligned}
$$

by Lemma 1 . Setting

$$
\bar{\ell}^{t}=\frac{3}{2 t} \frac{\zeta_{2}}{\zeta_{3}}
$$

as in [21] and using Lemma 3, we conclude the assertion.

Now we consider the survival function of the conditional length distribution of the typical I-segment of $Y(t)$, given its direction $u \in \delta_{+}^{2}$, i.e.

$$
H_{\ell \mid \varphi=u}^{t}(x)=\int \mathbf{1}\{\operatorname{length}(L)>x\} \mathbb{D}_{\varphi(L)=u}^{Y(t)}(\mathrm{d} L),
$$

where $\mathbb{D}_{\varphi(L)=u}^{Y(t)}(\mathrm{d} L)$ denotes the respective conditional distribution of the typical I-segment. The length-weighted case $\widetilde{H}_{\ell \mid \varphi=u}^{t}$ is defined analogously. The two distributional identities in Lemma 1 and Lemma 4 together with Lemma 2 imply the following result.

Lemma 5. The length distribution of the typical I-segment of $Y(t)$, given its direction $u \in 8_{+}^{2}$, has survival function

$$
H_{\ell \mid \varphi=u}^{t}(x)=\int_{0}^{t} \frac{3 s^{2}}{t^{3}} \mathrm{e}^{-\Lambda([u]) s x} \mathrm{~d} s,
$$

and the length distribution of the length-weighted typical I-segment of $Y(t)$, given its direction $u \in 8_{+}^{2}$, has survival function

$$
\widetilde{H}_{\ell \mid \varphi=u}^{t}(x)=\int_{0}^{t} \frac{2 s}{t^{2}}(1+\Lambda([u]) s x) \mathrm{e}^{-\Lambda([u]) s x} \mathrm{~d} s .
$$

\subsection{Length-weighted mark distributions}

For construction times $0<r<s$, we consider the states $Y(r)$ and $Y(s)$, and introduce three different random sets of unions of I-segments of $Y(s)$ :

- the union of all I-segments of $Y(s)$,

- the union of all I-segments of $Y(s)$ with birth times in $(r, s]$ that appear in the interior of the cells of $Y(r)$, 
- the union of I-segments of $Y(s)$ with birth times in $(r, s]$ that appear on the facets of the cells of $Y(r)$.

For these random sets, the corresponding length measures, the length intensities, and the directional distributions in the typical edge point (the length-weighted case) are respectively denoted by

- $\mu(s, \cdot), L_{V}(s)=\mathrm{E} \mu\left(s,[0,1]^{3}\right)$, and $\widetilde{\mathbb{Q}}_{\varphi}^{s}$,

- $\mu^{*}(s-r, \cdot), L_{V}^{*}(s-r)=\mathrm{E} \mu^{*}\left(s-r,[0,1]^{3}\right)$, and $\widetilde{\mathbb{Q}}_{\varphi}^{*, s-r}$,

- $\mu(r, s, \cdot), L_{V}(r, s)=\mathrm{E} \mu\left(r, s,[0,1]^{3}\right)$, and $\tilde{\mathbb{Q}}_{\varphi}^{r, s}$.

For a Borel set $B \subset \mathbb{R}^{3}$, the value $\mu^{*}(s-r, B)$ for example is the total length of edges of $Y(s)$ with birth times in $(r, s]$, which are located in the interior of the cells of $Y(r)$ and in $B$. It is evident that, for $0<r<s$, we have

$$
\mu(s, \cdot)=\mu(r, \cdot)+\mu(r, s, \cdot)+\mu^{*}(s-r, \cdot)
$$

for the length measures. From the STIT property, the following relations can be deduced.

Lemma 6. We have $L_{V}^{*}(s-r)=\underset{\widetilde{\widetilde{N}}}{=} L_{V}(s-r)$ and $L_{V}(s)=\zeta_{2} s^{2}$ with $\zeta_{2}$ given by (4). Furthermore, $\widetilde{\mathbb{Q}}_{\varphi}^{*, s-r}=\widetilde{\mathbb{Q}}_{\varphi}^{s-r}=\widetilde{\mathbb{Q}}_{\varphi}^{s}=\widetilde{\mathcal{R}}$ with $\widetilde{\mathcal{R}}$ defined via $(5)$.

Proof. Resorting to (2), we find that

$$
L_{V}^{*}(s-r)=L_{V}(s-r) \text { and } \quad \widetilde{\mathbb{Q}}_{\varphi}^{*, s-r}=\widetilde{\mathbb{Q}}_{\varphi}^{s-r} .
$$

The relation $L_{V}(s)=\zeta_{2} s^{2}$ is Equation (8) of [13] and the fact that $\widetilde{\mathbb{Q}}_{\varphi}^{s}$ does not depend on $s$ follows from [13, Equation (14)]. The remaining equality $\widetilde{\mathbb{Q}}_{\varphi}^{s}=\widetilde{\mathcal{R}}$ is a consequence of [13, Equation (11)]. This completes the proof.

In a next step, for the STIT tessellation $Y(t)$ with $t>0$, we consider the joint length-weighted distribution $\widetilde{\mathbb{Q}}_{\varphi, \beta, \beta_{\text {carr }}}^{t}$ of direction $\varphi \in 8_{+}^{2}$ and birth times $\beta, \beta_{\text {carr }} \in(0, t]$.

Lemma 7. For any Borel set $U \subset 8_{+}^{2}$ and $0<r<s<t$, we have

$$
\widetilde{\mathbb{Q}}_{\varphi, \beta, \beta_{\text {carr }}}^{t}(U \times(r, s] \times(0, r])=\frac{2\left(r s-r^{2}\right)}{t^{2}} \widetilde{\mathcal{R}}(U) .
$$

The corresponding joint density of $\left(\beta, \beta_{\mathrm{carr}}\right)$ with respect to the Lebesgue measure on $(0, t)^{2}$ is

$$
\tilde{p}_{\beta, \beta_{\text {carr }}}(s, r)=\frac{2}{t^{2}} \mathbf{1}\{0<r<s<t\},
$$

i.e. $\varphi$ and $\left(\beta, \beta_{\text {carr }}\right)$ are independent and $\left(\beta, \beta_{\text {carr }}\right)$ is uniformly distributed on the triangle $\left\{(s, r) \in \mathbb{R}^{2}: 0<r<s<t\right\}$.

Proof. We use (9) to conclude that

$$
L_{V}(s)=L_{V}(r)+L_{V}^{*}(s-r)+L_{V}(r, s)
$$

for the length intensities defined above. In view of Lemma 6 we obtain, from (10),

$$
L_{V}(r, s)=2 \zeta_{2}\left(r s-r^{2}\right)
$$


From the definition of the length-weighted directional distribution, it follows that the mean total length per unit volume of I-segments of $Y(t)$ with direction in a Borel set $U \subset 8_{+}^{2}$ is $L_{V}(t) \widetilde{\mathcal{R}}(U)$. Combining this with (10) and (11) yields

$$
L_{V}(r, s) \widetilde{\mathcal{R}}(U)=2 \zeta_{2}\left(r s-r^{2}\right) \widetilde{\mathcal{R}}(U) .
$$

Thus, for the mark distribution in a typical edge point, we find that

$$
\widetilde{\mathbb{Q}}_{\varphi, \beta, \beta_{\text {carr }}}^{t}(U \times(r, s] \times(0, r])=\frac{L_{V}(r, s) \widetilde{\mathcal{R}}(U)}{L_{V}(t)}=\frac{2\left(r s-r^{2}\right)}{t^{2}} \widetilde{\mathcal{R}}(U),
$$

and, hence, $\varphi$ and $\left(\beta, \beta_{\text {carr }}\right)$ are independent. Since $\beta_{\text {carr }}<\beta$ with probability 1 , we have, for the joint distribution function $\widetilde{F}_{\beta, \beta_{\text {carr }}}(s, r)$ of $\beta$ and $\beta_{\text {carr }}$ with $0<r<s<t$,

$$
\widetilde{\mathbb{Q}}_{\beta, \beta_{\text {carr }}}^{t}((r, s] \times(0, r])=\widetilde{F}_{\beta, \beta_{\text {carr }}}(s, r)-\widetilde{\mathbb{Q}}_{\beta, \beta_{\text {carr }}}^{t}((0, r] \times(0, r]),
$$

where

$$
\widetilde{\mathbb{Q}}_{\beta, \beta_{\text {carr }}}^{t}((r, s] \times(0, r])=\widetilde{\mathbb{Q}}_{\varphi, \beta, \beta_{\text {carr }}}^{t}\left(8_{+}^{2} \times(r, s] \times(0, r]\right)
$$

stands for the joint distribution of $\left(\beta, \beta_{\text {carr }}\right)$ in a typical edge point of $Y(t)$. Partial differentiation with respect to $r$ and then with respect to $s$ yields $\widetilde{p}_{\beta, \beta_{\text {carr }}}(s, r)$ (note that the $\widetilde{\mathbb{Q}}$-term on the right-hand side of (12) does not depend on $s$ and, thus, vanishes after differentiation with respect to $s$ ), completing the proof.

We now turn to the joint length-weighted distribution $\widetilde{\mathbb{Q}}_{\ell, \varphi, \beta, \beta_{\text {carr }}}^{t}$ of the length, direction, and birth times of the I-segment, i.e. of the I-segment through a typical edge point of $Y(t)$. The key is the conditional length distribution of the I-segment, given its direction $u \in 8_{+}^{2}$. For any point $z$ in the edge skeleton of $Y(t)$, denote by $\ell(z), \varphi(z), \beta(z)$, and $\beta_{\text {carr }}(z)$ the almost surely uniquely determined mark of the I-segment through $z$. According to the definition of the mark distribution (see [15, p. 84]) we have, for any Borel set $U \subset 8_{+}^{2}$, using the Campbell theorem and (9),

$$
\begin{aligned}
& \left.\widetilde{\mathbb{Q}}_{\ell, \varphi, \beta, \beta_{\text {carr }}}^{t}(x, \infty) \times U \times(r, s] \times(0, r]\right) \\
& =\frac{1}{L_{V}(t)} \mathrm{E} \int \mathbf{1}\{\ell(z)>x, \varphi(z) \in U\} \mu(r, s, \mathrm{~d} z) \\
& =\frac{1}{L_{V}(t)} \mathrm{E}\left[\int \mathbf{1}\{\ell(z)>x, \varphi(z) \in U\} \mu(s, \mathrm{~d} z)-\int \mathbf{1}\{\ell(z)>x, \varphi(z) \in U\} \mu(r, \mathrm{~d} z)\right. \\
& \left.\quad-\int \mathbf{1}\{\ell(z)>x, \varphi(z) \in U\} \mu^{*}(s-r, \mathrm{~d} z)\right] \\
& =\frac{1}{L_{V}(t)}\left[L_{V}(s) \widetilde{\mathbb{Q}}_{\ell, \varphi}^{s}((x, \infty) \times U)-L_{V}(r) \widetilde{\mathbb{Q}}_{\ell, \varphi}^{r}((x, \infty) \times U)\right. \\
& \left.\quad-L_{V}(s-r) \widetilde{\mathbb{Q}}_{\ell, \varphi}^{*, s-r}((x, \infty) \times U)\right],
\end{aligned}
$$

where $\widetilde{\mathbb{Q}}_{\ell, \varphi}^{*, s-r}$ is the $(\ell, \varphi)$-marginal distribution of $\widetilde{\mathbb{Q}}_{\ell, \varphi, \beta, \beta_{\text {carr }}}^{*, s-r}$. Write $\widetilde{\mathbb{Q}}_{\ell \mid \varphi=u}^{s}$ for the lengthweighted conditional distribution of the length under the condition $\varphi=u$. Then

$$
\begin{aligned}
\widetilde{\mathbb{Q}}_{\ell, \varphi}^{s}((x, \infty) \times U) & =\int \mathbf{1}\{l>x, u \in U\} \widetilde{\mathbb{Q}}_{\ell, \varphi}^{s}(\mathrm{~d}(l, u)) \\
& =\iint \mathbf{1}\{l>x\} \widetilde{\mathbb{Q}}_{\ell \mid \varphi=u}^{s}(\mathrm{~d} l) \mathbf{1}\{u \in U\} \widetilde{\mathcal{R}}(\mathrm{d} u),
\end{aligned}
$$


where we have used Lemma 6. In view of (13) and (14) we now determine certain conditional length distributions.

Lemma 8. For $0<r<s, 0<x$, and a Borel set $U \subset 8_{+}^{2}$, we have

$$
\begin{aligned}
& L_{V}(s) \widetilde{\mathbb{Q}}_{\ell, \varphi}^{s}((x, \infty) \times U)-L_{V}(r) \widetilde{\mathbb{Q}}_{\ell, \varphi}^{r}((x, \infty) \times U) \\
&=\zeta_{2} \int_{U} \frac{2}{\Lambda([u])^{2} x^{2}}\left([3+\Lambda([u]) r x(3+\Lambda([u]) r x)] \mathrm{e}^{-\Lambda([u]) r x}\right. \\
&\left.\quad-[3+\Lambda([u]) s x(3+\Lambda([u]) s x)] \mathrm{e}^{-\Lambda([u]) s x}\right) \widetilde{\mathcal{R}}(\mathrm{d} u) .
\end{aligned}
$$

Proof. We start by recalling from Lemma 6 that $L_{V}(s)=\zeta_{2} s^{2}$ and $L_{V}(r)=\zeta_{2} r^{2}$. Using (14) and the integral representation for the conditional survival function provided in Lemma 5 , we obtain

$$
\begin{aligned}
L_{V}(s) & \widetilde{\mathbb{Q}}_{\ell, \varphi}^{s}((x, \infty) \times U)-L_{V}(r) \widetilde{\mathbb{Q}}_{\ell, \varphi}^{r}((x, \infty) \times U) \\
& =\zeta_{2} s^{2} \int_{U} \widetilde{H}_{\ell \mid \varphi=u}^{s}(x) \widetilde{\mathcal{R}}(\mathrm{d} u)-\zeta_{2} r^{2} \int_{U} \widetilde{H}_{\ell \mid \varphi=u}^{r}(x) \widetilde{\mathcal{R}}(\mathrm{d} u) \\
& =2 \zeta_{2} \int_{U} \int_{r}^{s} v(1+\Lambda([u]) v x) \mathrm{e}^{-\Lambda([u]) v x} \mathrm{~d} v \widetilde{\mathcal{R}}(\mathrm{d} u) ;
\end{aligned}
$$

integration yields the claim.

It remains to determine the last item $\widetilde{\mathbb{Q}}_{\ell, \varphi}^{*, s-r}((x, \infty) \times U)$ in $(13)$, which in view of Lemma 6 may be written in the form

$$
\widetilde{\mathbb{Q}}_{\ell, \varphi}^{*, s-r}((x, \infty) \times U)=\iint \mathbf{1}\{l>x\} \widetilde{\mathbb{Q}}_{\ell \mid \varphi=u}^{*, s-r}(\mathrm{~d} l) \mathbf{1}\{u \in U\} \widetilde{R}(\mathrm{~d} u) .
$$

Lemma 9. For $0<r<s, 0<x$, and a Borel set $U \subset 8_{+}^{2}$, we have

$$
\begin{aligned}
& L_{V}(s-r) \tilde{\mathbb{Q}}_{\ell, \varphi}^{*, s-r}((x, \infty) \times U) \\
& \quad=\zeta_{2} \int_{U} \frac{2}{\Lambda([u])^{2} x^{2}}\left(3\left(\mathrm{e}^{-\Lambda([u]) r x}-\mathrm{e}^{-\Lambda([u]) s x}\right)\right. \\
& \left.\quad+\Lambda([u])^{2} s x^{2}\left(r \mathrm{e}^{-\Lambda([u]) r x}-s \mathrm{e}^{-\Lambda([u]) s x}\right)\right) \widetilde{\mathcal{R}}(\mathrm{d} u) .
\end{aligned}
$$

Proof. To derive the result, we make use of a method developed for the planar case in [8] and consider the conditional survival function $\widetilde{G}_{\ell \mid \varphi=u}^{*, s-r}$ of the length of the remaining I-segment, that is, the part of the I-segment that lies above a random point of the edge skeleton, which is selected according to $\mu^{*}(s-r, \cdot)$. (In this proof, the letter $G$ will always refer to a remaining I-segment while $H$ refers to the whole I-segment.) Regarding (2), the distribution of the remaining length is the distribution of the minimum of the remaining length in $Y(s-r)$ and the distance (in direction $u$ ) to the 'frame' tessellation $Y(r)$, where the I-segment is cut. Owing to (P6), the latter has conditional survival function $\mathrm{e}^{-\Lambda([u]) r x}$. Making use of the independence of $Y(r)$ and $Y(s-r)$, we obtain

$$
\widetilde{G}_{\ell \mid \varphi=u}^{*, s-r}(x)=\widetilde{G}_{\ell \mid \varphi=u}^{s-r}(x) \mathrm{e}^{-\Lambda([u]) r x},
$$

where $\widetilde{G}_{\ell \mid \varphi=u}^{s-r}$ is the corresponding conditional survival function for the remaining I-segment of the tessellation $Y(s-r)$. It follows from the Palm theory (compare with Equation (14) of [8]) 
that the survival function of the length of the remaining I-segment as considered above and the conditional survival function $H_{\ell \mid \varphi=u}^{s-r}$ of the length of the (whole) typical I-segment are related by

$$
\widetilde{G}_{\ell \mid \varphi=u}^{s-r}(x)=\frac{1}{\bar{\ell}_{\varphi=u}^{s-r}} \int_{x}^{\infty} H_{\ell \mid \varphi=u}^{s-r}(a) \mathrm{d} a,
$$

where $\bar{\ell}_{\varphi=u}^{s-r}$ is the conditional mean length of the typical I-segment in $Y(s-r)$. Using Lemma 5, we obtain $\bar{\ell}_{\varphi=u}^{s-r}=3 / 2 \Lambda([u])(s-r)$. Combining (16) with (17) and again using Lemma 5, we find that

$$
\widetilde{G}_{\ell \mid \varphi=u}^{*, s-r}(x)=\frac{2 \Lambda([u])}{(s-r)^{2}} \mathrm{e}^{-\Lambda([u]) r x} \int_{x}^{\infty} \int_{0}^{s-r} v^{2} \mathrm{e}^{-\Lambda([u]) v a} \mathrm{~d} v \mathrm{~d} a .
$$

Once more we use the Palm theory developed in [8] (see, in particular, Equation (16) therein) to conclude that the survival function $H_{\ell \mid \varphi=u}^{*, s-r}$ of the conditional length distribution of the corresponding typical I-segment equals

$$
H_{\ell \mid \varphi=u}^{*, s-r}(x)=\left(\lim _{x \downarrow 0} \frac{\partial \widetilde{G}_{\ell \mid \varphi=u}^{*, s-r}(x)}{\partial x}\right)^{-1} \frac{\partial \widetilde{G}_{\ell \mid \varphi=u}^{*, s-r}(x)}{\partial x},
$$

and, thus, we find that

$$
\begin{aligned}
H_{\ell \mid \varphi=u}^{*, s-r}(x)= & \frac{6}{\Lambda([u])^{3}(s-r)^{2}(2 s+r) x^{3}} \\
& \times\left((2+\Lambda([u]) r x) \mathrm{e}^{-\Lambda([u]) r x}\right. \\
& \left.\quad-\left(2+(2 s-r) \Lambda([u]) x+\Lambda([u])^{2} s(s-r) x^{2}\right) \mathrm{e}^{-\Lambda([u]) s x}\right)
\end{aligned}
$$

from (18) by integration. Using this formula, we calculate the corresponding mean I-segment length $\bar{\ell}_{\varphi=u}^{*, s-r}$ as

$$
\bar{\ell}_{\varphi=u}^{*, s-r}=\int_{0}^{\infty} H_{\ell \mid \varphi=u}^{*, s-r}(x) \mathrm{d} x=\frac{3}{\Lambda([u])(2 s+r)},
$$

and, again by length weighting, we obtain the corresponding survival function

$$
\widetilde{H}_{\ell \mid \varphi=u}^{*, s-r}(x)=\widetilde{\mathbb{Q}}_{\ell \mid \varphi=u}^{*, s-r}((x, \infty))=\int_{x}^{\infty} \frac{z}{\bar{\ell}_{\varphi=u}^{*, s-r}} \frac{\partial H_{\ell \mid \varphi=u}^{*, s-r}(z)}{\partial z} \mathrm{~d} z,
$$

which is given by

$$
\begin{aligned}
\widetilde{H}_{\ell \mid \varphi=u}^{*, s-r}(x)= & \frac{2}{\Lambda([u])^{2}(s-r)^{2} x^{2}} \\
& \times\left(3\left(\mathrm{e}^{-\Lambda([u]) r x}-\mathrm{e}^{-\Lambda([u]) s x}\right)+\Lambda([u])^{2} s x^{2}\left(r \mathrm{e}^{-\Lambda([u]) r x}-s \mathrm{e}^{-\Lambda([u]) s x}\right)\right) .
\end{aligned}
$$

Taking into account the factor $L_{V}(s-r)=\zeta_{2}(s-r)^{2}$, we complete the proof by using (15).

Combining (13) with Lemma 8, Lemma 9, and the fact that $L_{V}(t)=\zeta_{2} t^{2}$ from Lemma 6 , we arrive at

$$
\begin{aligned}
\left.\widetilde{\mathbb{Q}}_{\ell, \varphi, \beta, \beta_{\text {carr }}}^{t}(x, \infty) \times U \times(r, s] \times(0, r]\right) \\
\quad=\int_{U} \frac{2 r}{\Lambda([u]) x t^{2}}\left((2+\Lambda([u]) r x) \mathrm{e}^{-\Lambda([u]) r x}-(2+\Lambda([u]) s x) \mathrm{e}^{-\Lambda([u]) s x}\right) \widetilde{\mathcal{R}}(\mathrm{d} u) .
\end{aligned}
$$


A relation similar to (12) for $\widetilde{\mathbb{Q}}_{\ell, \varphi, \beta, \beta_{\text {carr }}}^{t}$ and the respective distribution function yields, after differentiation with respect to $r, s$, and $x$, the following assertion.

Lemma 10. The conditional joint density of length $\ell$ and the birth time vector $\left(\beta, \beta_{\text {carr }}\right)$ of the length-weighted typical I-segment in $Y(t)$, given its direction $\varphi=u \in 8_{+}^{2}$, equals

$$
\tilde{p}_{\ell, \beta, \beta_{\text {carr }} \mid \varphi=u}(x, s, r)=\frac{2 \Lambda([u])^{2} s^{2}}{t^{2}} x \mathrm{e}^{-\Lambda([u]) s x} \mathbf{1}\{x>0\} \mathbf{1}\{0<r<s<t\} .
$$

We now turn to the proofs of our main results. In fact, Lemma 10 is key for the proof of Theorem 1, which forms the basis for deriving Theorems 2 and 3.

\subsection{Proofs of the main results}

Proof of Theorem 1. (i) Apply Lemma 1 with $f(L)=\mathbf{1}\{\varphi(L) \in U\}$ for a measurable $U \subset 8_{+}^{2}$, where $\varphi(L)$ is the direction of $L$. Since, for homogeneous Poisson plane tessellations with intensity measure $s \Lambda, s>0$, the directional distribution of the typical edge is invariant with respect to the scaling factor $s$, the distribution of the direction of the typical I-segment of $Y(t)$ is the same as the directional distribution of the typical edge in a Poisson plane tessellation with intensity measure $t \Lambda$. Thus, we can apply Theorem 1 of [6], which immediately yields assertion (i).

(ii) The key is the relation

$$
p_{\ell, \beta, \beta_{\text {carr }} \mid \varphi=u}(x, s, r)=\frac{\bar{\ell}_{\varphi=u}^{t}}{x} \widetilde{p}_{\ell, \beta, \beta_{\text {carr }} \mid \varphi=u}(x, s, r),
$$

where $\bar{\ell}_{\varphi=u}^{t}$ is the conditional mean length of the typical I-segment in $Y(t)$, given $\varphi=u$. With Lemma 5 we calculate $\bar{\ell}_{\varphi=u}^{t}=3 / 2 t \Lambda([u])$, and Lemma 10 implies that

$$
\begin{aligned}
p_{\ell, \beta, \beta_{\text {carr }} \mid \varphi=u}(x, s, r) & =\frac{3}{2 \Lambda([u]) t x} \frac{2 \Lambda([u])^{2} s^{2}}{t^{2}} x \mathrm{e}^{-\Lambda([u]) x} \mathbf{1}\{x>0\} \\
& =\frac{3 s}{t^{3}} \Lambda([u]) s \mathrm{e}^{-\Lambda([u]) s x} \mathbf{1}\{x>0\} .
\end{aligned}
$$

Integration with respect to $x$ yields

$$
p_{\beta, \beta_{\text {carr }} \mid \varphi=u}(s, r)=\frac{3 s}{t^{3}} \int_{0}^{\infty} \Lambda([u]) s \mathrm{e}^{-\Lambda([u]) s x} \mathrm{~d} x=\frac{3 s}{t^{3}}, \quad 0<r<s<t .
$$

Since it does not depend on $u$, we have $p_{\beta, \beta_{\text {carr }}}=p_{\beta, \beta_{\text {carr }} \mid \varphi=u}$ and this completes the proof of (ii).

(iii) Similarly to the proof of part (ii), we use the following relation for the conditional densities:

$$
p_{\ell \mid \varphi=u, \beta=s, \beta_{\text {carr }}=r}(x)=\frac{\bar{\ell}_{\varphi=u, \beta=s, \beta_{\text {carr }}^{t}=r}}{x} \tilde{p}_{\ell \mid \varphi=u, \beta=s, \beta_{\text {carr }=r}}(x) .
$$

Here $\bar{\ell}_{\varphi=u, \beta=s, \beta_{\text {carr }}=r}$ is the conditional mean length of the typical I-segment in $Y(t)$, given $\varphi$, $\beta$, and $\beta_{\text {carr }}$. Combining (19) and (20) we obtain

$$
p_{\ell \mid \varphi=u, \beta=s, \beta_{\text {carr }}=r}(x)=\frac{p_{\ell, \beta, \beta_{\text {carr }} \mid \varphi=u}(x, s, r)}{p_{\beta, \beta_{\text {carr }} \mid \varphi=u}(s, r)}=\Lambda([u]) s \mathrm{e}^{-\Lambda([u]) s x} \mathbf{1}\{x>0\},
$$


which implies that

$$
\bar{\ell}_{\varphi=u, \beta=s, \beta_{\mathrm{carr}}=r}^{t}=\frac{1}{\Lambda([u]) s} .
$$

This completes the proof.

Proofs of Corollaries 1-3. The formulae in Corollary 1 follow by straightforward integration from Theorem 1(ii).

To prove Corollary 2, we first note that in the proof of Theorem 1(ii) we showed that

$$
p_{\ell, \beta, \beta_{\text {carr }} \mid \varphi=u}(x, s, r)=\frac{3 s}{t^{3}} \Lambda([u]) s \mathrm{e}^{-\Lambda([u]) s x},
$$

which proves the formula. Integration with respect to $x$ yields the conditional joint density of the birth time vector, namely,

$$
p_{\beta, \beta_{\mathrm{carr}} \mid \varphi=u}(s, r)=\frac{3 s}{t^{3}} .
$$

This shows the independence of $\varphi$ and the birth times $\left(\beta, \beta_{\text {carr }}\right)$, completing the proof of Corollary 2.

The statement of Corollary 3 follows by integration of (19).

Proof of Theorem 2. The first step is to verify the following expression for $\mathrm{p}_{n}$ :

$$
\begin{gathered}
\mathrm{p}_{n}=\int_{0}^{t} \int_{0}^{s} \int_{\delta_{+}^{2}} \int_{0}^{\infty} \frac{3 \Lambda([u]) s^{2}}{t^{3}} \mathrm{e}^{-s \Lambda([u]) x} \frac{(\Lambda([u]) x(3 t-2 s-r))^{n}}{n !} \\
\times \mathrm{e}^{-\Lambda([u]) x(3 t-2 s-r)} \mathrm{d} x \mathcal{R}_{\mathrm{typ}}(\mathrm{d} u) \mathrm{d} r \mathrm{~d} s .
\end{gathered}
$$

We start by noting that an I-segment arises as the intersection of two I-polygons, which are born at different time instants, the maximum of which is the birth time of the I-segment and has a density $p_{\beta}(s)$ given by (7). However, in contrast to the planar case, I-segments in a spatial STIT tessellation can have vertices in their relative interior at their time of birth. These vertices are generated by extant I-segments 'on the backside' of the carrying polygon; see the left diagram of Figure 2. Given its length $\ell=x \in(0, \infty)$, its direction $\varphi=u \in s_{+}^{2}$, its time of birth $\beta=s \in(0, t)$, and the birth time $\beta_{\text {carr }}=r \in(0, s)$ of the carrying I-polygon, the number $N_{\text {birth }}$ of vertices in the relative interior of the typical I-segment at birth time $s$ has, by (2) and (P6), a Poisson distribution with parameter $\Lambda([u]) x(s-r)$. Moreover, in the time interval $(s, t]$, on both sides of the I-polygon with birth time $s$ and 'behind' the carrying I-polygon a Poisson-distributed number of vertices appear in the relative interior of the segment, whose parameter is, again by (2) and (P6), $\Lambda([u]) x(t-s)$; see the right diagram of Figure 2. Thus, the sum of these independent numbers is again Poisson distributed, but with parameter $3 \Lambda([u]) x(t-s)$. Adding the independent number $N_{\text {birth }}$ leads to a Poisson distribution with parameter $\Lambda([u]) x(3 t-2 s-r)$.

Recall that in Corollary 2 we calculated the conditional joint density of the length and birth time vector, and, thus, mixing the Poisson-distributed number of points with respect to this density and the directional distribution $\mathcal{R}_{\text {typ }}$, we arrive at (21).

We now show that (21) is equivalent to (8). First, using

$$
\int_{0}^{\infty}(\Lambda([u]) x)^{n} \mathrm{e}^{-\Lambda([u]) s x} \mathrm{e}^{-\Lambda([u]) x(3 t-2 s-r)} \mathrm{d} x=\frac{n !}{\Lambda([u])(3 t-s-r)^{n+1}},
$$


we see that (21) can be transformed into

$$
\mathrm{p}_{n}=\int_{0}^{t} \int_{0}^{s} \int_{\delta_{+}^{2}} \frac{3 s^{2}}{t^{3}} \frac{(3 t-2 s-r)^{n}}{(3 t-s-r)^{n+1}} \mathcal{R}_{\mathrm{typ}}(\mathrm{d} u) \mathrm{d} r \mathrm{~d} s=\int_{0}^{t} \int_{0}^{s} \frac{3 s^{2}}{t^{3}} \frac{(3 t-2 s-r)^{n}}{(3 t-s-r)^{n+1}} \mathrm{~d} r \mathrm{~d} s .
$$

Applying the substitution $b=1-r / s$ to the inner integral yields

$$
\mathrm{p}_{n}=\int_{0}^{t} \int_{0}^{1} \frac{3 s^{3}}{t^{3}} \frac{(3 t-s(3-b))^{n}}{(3 t-s(2-b))^{n+1}} \mathrm{~d} b \mathrm{~d} s
$$

and applying the similar substitution $a=1-s / t$, leads to (8). This completes the proof.

Proof of Corollary 4. The numbers shown in Corollary 4 can immediately be calculated from the explicit formula in Theorem 2. To see this, write

$$
\begin{aligned}
\sum_{n=0}^{\infty} n \mathrm{p}_{n} & =3 \int_{0}^{1} \int_{0}^{1}(1-a)^{3} \sum_{n=0}^{\infty} n \frac{(3-(1-a)(3-b))^{n}}{(3-(1-a)(2-b))^{n+1}} \mathrm{~d} b \mathrm{~d} a \\
& =3 \int_{0}^{1} \int_{0}^{1}(1-a)(a(3-b)+b) \mathrm{d} b \mathrm{~d} a \\
& =2
\end{aligned}
$$

and, similarly,

$$
\begin{aligned}
\sum_{n=0}^{\infty} n^{2} \mathrm{p}_{n} & =3 \int_{0}^{1} \int_{0}^{1}(1-a)^{3} \sum_{n=0}^{\infty} n^{2} \frac{(3-(1-a)(3-b))^{n}}{(3-(1-a)(2-b))^{n+1}} \mathrm{~d} b \mathrm{~d} a \\
& =3 \int_{0}^{1} \int_{0}^{1} a^{2}\left(2 b^{2}-11 b+15\right)-a\left(4 b^{2}-10 b-3\right)+b(1+2 b) \mathrm{d} a \mathrm{~d} b \\
& =\frac{71}{3}
\end{aligned}
$$

which yields the variance value $\frac{71}{3}-2^{2}=\frac{59}{3}$.

Proof of Theorem 3. Let the birth time $\beta=s<t$ of the typical I-segment and the birth time $\beta_{\text {carr }}=r<s$ of the carrying I-polygon be given. At time $s$, the typical I-segment can have only X-type vertices in its relative interior (this is the number $N_{\text {birth }}$ in the proof of Theorem 2) and further vertices of this type can only be created from 'behind' the segment until time $t$. At time $s$ the I-segment is an edge (1-facet) of two cells. Vertices of type T in the interior of the I-segment can appear in the time interval $(s, t)$ only by further division of these two adjacent cells. The proof now readily follows the lines of the proof of Theorem 2 and for this reason the details are omitted.

Proof of Corollary 5. Corollary 5 follows from Theorem 3 by direct calculation. For example, the mean number of T-type vertices in the relative interior of the typical I-segment can be calculated as follows:

$$
\begin{aligned}
\sum_{m=0}^{\infty} m \sum_{n=0}^{\infty} \mathrm{p}_{m, n} & =3 \sum_{m=0}^{\infty} m \int_{0}^{1} \int_{0}^{1} \frac{(1-a)^{3}}{1+a}\left(\frac{2 a}{1+a}\right)^{m} \mathrm{~d} b \mathrm{~d} a \\
& =6 \int_{0}^{1} a(1-a) \mathrm{d} a \int_{0}^{1} \mathrm{~d} b \\
& =1 .
\end{aligned}
$$


For the second moment, we find that

$$
\begin{aligned}
\sum_{m=0}^{\infty} m^{2} \sum_{n=0}^{\infty} \mathrm{p}_{m, n} & =3 \sum_{m=0}^{\infty} m^{2} \int_{0}^{1} \int_{0}^{1} \frac{(1-a)^{3}}{1+a}\left(\frac{2 a}{1+a}\right)^{m} \mathrm{~d} b \mathrm{~d} a \\
& =6 \int_{0}^{1} a(1+3 a) \mathrm{d} a \int_{0}^{1} \mathrm{~d} b \\
& =9,
\end{aligned}
$$

so that the variance equals $9-1^{2}=8$. Similar calculations yield mean 1 and in particular variance $\frac{11}{3}$ for the number of $\mathrm{X}$-vertices in the relative interior of the typical I-segment. Analogously, the joint mean number of $\mathrm{T}$ - and $\mathrm{X}$-vertices is

$$
\sum_{m=0}^{\infty} m \sum_{n=0}^{\infty} n \mathrm{p}_{m, n}=12 \int_{0}^{1} \int_{0}^{1} a(a+b-a b) \mathrm{d} b \mathrm{~d} a=5 .
$$

Consequently, the covariance equals 4 , which completes the proof.

\section{Acknowledgements}

The authors would like to thank Claudia Redenbach (Kaiserslautern) for providing the simulations in Figure 1. We also thank an anonymous referee for his or her valuable comments. VW and WN acknowledge support from the DFG, grant numbers WE 1899/3-1 and NA 247/6-1.

\section{References}

[1] Chen, F. K. C. And Cowan, R. (1999). Invariant distributions for shapes in sequences of randomly-divided rectangles. Adv. Appl. Prob. 31, 1-14.

[2] Cowan, R. (1997). Shapes of rectangular prisms after repeated random division. Adv. Appl. Prob. 29, 26-37.

[3] Cowan, R. (2010). New classes of random tessellations arising from iterative division of cells. Adv. Appl. Prob. 42, 26-47.

[4] Heinrich, L. (2009). Central limit theorems for motion-invariant Poisson hyperplanes in expanding convex bodies. Rend. Circ. Mat. Palermo Ser. II Suppl. 81, 187-212.

[5] Heinrich, L. And Muche, L. (2008). Second-order properties of the point process of nodes in a stationary Voronoi tessellation. Math. Nachr. 281, 350-375.

[6] Hug, D. And Schneider, R. (2011). Faces with given directions in anisotropic Poisson hyperplane mosaics. Adv. Appl. Prob. 43, 308-321.

[7] LachièZe-Rey, R. (2011). Mixing properties for STIT tessellations. Adv. Appl. Prob. 43, 40-48.

[8] Mecke, J., Nagel, W. ANd Weiss, V. (2007). Length distributions of edges in planar stationary and isotropic STIT tessellations. J. Contemp. Math. Anal. 42, $28-43$.

[9] Mecke, J., Nagel, W. and Weiss, V. (2008). A global construction of homogeneous random planar tessellations that are stable under iteration. Stochastics 80, 51-67.

[10] Mecke, J., NAgel, W. ANd Weiss, V. (2011). Some distributions for I-segments of planar random homogeneous STIT tessellations. Math. Nachr. 284, 1483-1495.

[11] Miles, R. E. AND Mackisack, M. S. (2002). A large class of random tessellations with the classic Poisson polygon distributions. Forma 17, 1-17.

[12] Nagel, W. AND WeIss, V. (2005). Crack STIT tessellations: characterization of stationary random tessellations stable with respect to iteration. Adv. Appl. Prob. 37, 859-883.

[13] Nagel, W. and Weiss, V. (2008). Mean values for homogeneous STIT tessellations in 3D. Image Anal. Stereology 27, 29-37.

[14] Redenbach, C. And ThäLe, C. (2011). Second-order comparison of three fundamental tessellation models. Statistics 21 pp. (electronic).

[15] Schneider, R. AND WeIL, W. (2008). Stochastic and Integral Geometry. Springer, Berlin.

[16] Schreiber, T. AND ThäLe, C. (2010). Second-order properties and central limit theory for the vertex process of iteration infinitely divisible and iteration stable random tessellations in the plane. Adv. Appl. Prob. 42, 913-935. 
[17] Schreiber, T. AND ThäLE, C. (2011). Intrinsic volumes of the maximal polytope process in higher dimensional STIT tessellations. Stoch. Process. Appl. 121, 989-1012.

[18] Schreiber, T. AND ThäLe, C. (2012). Geometry of iteration stable tessellations: connection with Poisson hyperplanes. Bernoulli 19pp. (electronic).

[19] Stoyan, D., Kendall, W. S. And Mecke, J. (1995). Stochastic Geometry and Its Applications, 2nd edn. John Wiley, Chichester.

[20] ThäLE, C. (2010). The distribution of the number of nodes in the relative interior of the typical I-segment in homogeneous planar anisotropic STIT tessellations. Comment. Math. Univ. Carolin. 51, 503-512.

[21] ThäLe, C. AND WeIss, V. (2010). New mean values for homogeneous spatial tessellations that are stable under iteration. Image Anal. Stereology 29, 143-157.

[22] ThäLe, C. AND WeIss, V. (2011). The combinatorial structure of spatial STIT tessellations. Preprint. Available at http://arxiv.org/abs/1111.0488v1.

[23] Weiss, V. and Cowan, R. (2011). Topological relationships in spatial tessellations. Adv. Appl. Prob. 43, $963-984$. 